\title{
A DENSITY RESULT IN VECTOR OPTIMIZATION
}

\author{
ALEXANDER J. ZASLAVSKI
}

Received 21 November 2005; Revised 2 March 2006; Accepted 12 March 2006

We study a class of vector minimization problems on a complete metric space such that all its bounded closed subsets are compact. We show that a subclass of minimization problems with a nonclosed set of minimal values is dense in the whole class of minimization problems.

Copyright (c) 2006 Hindawi Publishing Corporation. All rights reserved.

\section{Introduction and the main result}

The study of vector optimization problems has recently been a rapidly growing area of research. See, for example, [1-5] and the references mentioned therein. In this paper we study a class of vector minimization problems on a complete metric space such that all its bounded closed subsets are compact. This class of problems is associated with a complete metric space of continuous vector functions $\mathscr{A}$ defined below. For each $F$ from $\mathcal{A}$ we denote by $v(F)$ the set of all minimal elements of the image $F(X)=\{F(x): x \in X\}$. In this paper we will study the sets $v(F)$ with $F \in \mathscr{A}$. It is clear that for a minimization problem with only one criteria the set of minimal values is a singleton. In the present paper we will show that the subspace of all $F \in \mathscr{A}$ with nonclosed sets $v(F)$ is dense in $\mathscr{A}$. Therefore in general the sets $v(F), F \in \mathscr{A}$ can be rather complicated.

In this paper we use the convention that $\infty / \infty=1$ and denote by $\operatorname{Card}(E)$ the cardinality of the set $E$.

Let $\mathbb{R}$ be the set of real numbers and let $n$ be a natural number. Consider the finitedimensional space $R^{n}$ with the norm

$$
\|x\|=\left\|\left(x_{1}, \ldots, x_{n}\right)\right\|=\max \left\{\left|x_{i}\right|: i=1, \ldots, n\right\}, \quad x=\left(x_{1}, \ldots, x_{n}\right) \in R^{n}
$$

Let $\left\{e_{1}, \ldots, e_{n}\right\}$ be the standard basis in $R^{n}$ :

$$
e_{1}=(1,0, \ldots, 0), \ldots, e_{n}=(0, \ldots, 0,1)
$$

Hindawi Publishing Corporation International Journal of Mathematics and Mathematical Sciences Volume 2006, Article ID 29135, Pages 1-10

DOI 10.1155/IJMMS/2006/29135 
We equip the space $R^{n}$ with the natural order. Let $x=\left(x_{1}, \ldots, x_{n}\right), y=\left(y_{1}, \ldots, y_{n}\right) \in R^{n}$. We say that

$$
\begin{gathered}
x \geq y \quad \text { if } x_{i} \geq y_{i} \forall i \in\{1, \ldots, n\}, \\
x>y \quad \text { if } x \geq y, x \neq y, \\
x \gg y \quad \text { if } x_{i}>y_{i} \forall i \in\{1, \ldots, n\} .
\end{gathered}
$$

We say that $x \ll y$ (resp., $x<y, x \leq y)$ if $y \gg x$ (resp., $y>x, y \geq x$ ).

Let $(X, \rho)$ be a complete metric space such that each of its bounded closed subsets is compact. Fix $\theta \in X$.

Denote by $\mathscr{A}$ the set of all continuous mappings $F=\left(f_{1}, \ldots, f_{n}\right): X \rightarrow R^{n}$ such that for $i=1, \ldots, n$

$$
\lim _{\rho(x, \theta) \rightarrow \infty} f_{i}(x)=\infty
$$

For each $F=\left(f_{1}, \ldots, f_{n}\right), G=\left(g_{1}, \ldots, g_{n}\right) \in \mathscr{A}$ set

$$
\begin{gathered}
\tilde{d}(F, G)=\sup \left\{\left|f_{i}(x)-g_{i}(x)\right|: x \in X, i=1, \ldots, n\right\}, \\
d(F, G)=\tilde{d}(F, G)(1+\tilde{d}(F, G))^{-1} .
\end{gathered}
$$

Clearly the metric space $(\mathscr{A}, d)$ is complete.

Let $A \subset R^{n}$ be a nonempty set. An element $x \in A$ is called a minimal element of $A$ if there is no $y \in A$ for which $y<x$.

Let $F \in \mathscr{A}$. A point $x \in X$ is called a point of minimum of $F$ if $F(x)$ is a minimal element of $F(X)$. If $x \in X$ is a point of minimum of $F$, then $F(x)$ is called a minimal value of $F$. Denote by $M(F)$ the set of all points of minimum of $F$ and put $v(F)=F(M(F))$.

The following proposition is proved in [6].

Proposition 1.1. Let $F=\left(f_{1}, \ldots, f_{n}\right) \in \mathscr{A}$. Then $M(F)$ is a nonempty bounded subset of $(X, \rho)$ and for each $z \in F(X)$ there is $y \in v(F)$ such that $y \leq z$.

In the sequel we assume that $n \geq 2$ and that the space $(X, \rho)$ has no isolated points.

The following theorem is our main result. It will be proved in Section 2.

Theorem 1.2. Suppose that the space $(X, \rho)$ is connected. Let $F=\left(f_{1}, \ldots, f_{n}\right) \in \mathscr{A}$ and let $\epsilon>0$. Then there exists $G \in \mathscr{A}$ such that $\tilde{d}(F, G) \leq \epsilon$ and the set $v(G)$ is not closed.

\section{Proof of Theorem 1.2}

By Proposition 1.1 there exists $x_{*} \in X$ such that

$$
F\left(x_{*}\right) \in v(F) .
$$


There exists $\delta \in(0,1 / 4)$ such that

$$
\begin{gathered}
\left\|F(x)-F\left(x_{*}\right)\right\| \leq \frac{\epsilon}{8} \quad \text { for each } x \in X \text { such that } \rho\left(x, x_{*}\right) \leq 2 \delta, \\
\left\{z \in X: \rho\left(z, x_{*}\right)=8 \delta\right\} \neq \varnothing .
\end{gathered}
$$

Since the metric space $X$ is connected for each $t \in(0,8 \delta]$, there is $z \in X$ such that $\rho(z$, $\left.x_{*}\right)=t$.

It is clear that there exists a continuous function $\psi: X \rightarrow[0,1]$ such that

$$
\begin{array}{cc}
\psi(x)=1 & \text { for each } x \in X \text { satisfying } \rho\left(x, x_{*}\right) \leq \delta, \\
\psi(x)=0 & \text { for each } x \in X \text { satisfying } \rho\left(x, x_{*}\right) \geq 2 \delta .
\end{array}
$$

For $x \in X$ and $i=1, \ldots, n$ define

$$
\begin{gathered}
f_{i}^{(1)}(x)=\psi(x) f_{i}\left(x_{*}\right)+(1-\psi(x)) f_{i}(x), \\
F^{(1)}=\left(f_{1}^{(1)}, \ldots, f_{n}^{(1)}\right) .
\end{gathered}
$$

Clearly, $F^{(1)} \in \mathscr{A}$ and

$$
F^{(1)}(x)=F\left(x_{*}\right) \quad \forall x \in X \text { satisfying } \rho\left(x, x_{*}\right) \leq \delta .
$$

We will show that $\tilde{d}\left(F, F^{(1)}\right) \leq \epsilon / 8$. Let $x \in X$. If $\rho\left(x, x_{*}\right) \geq 2 \delta$, then $\psi(x)=0$ and $F^{(1)}(x)=$ $F(x)$. If $\rho\left(x, x_{*}\right) \leq 2 \delta$, then by $(2.2)$

$$
\left\|F(x)-F\left(x_{*}\right)\right\| \leq \frac{\epsilon}{8} .
$$

Combined with (2.5) the inequality above implies that

$$
\left\|F^{(1)}(x)-F(x)\right\|=\left\|\psi(x)\left(F\left(x_{*}\right)-F(x)\right)\right\| \leq\left\|F\left(x_{*}\right)-F(x)\right\| \leq \frac{\epsilon}{8} .
$$

Therefore

$$
\tilde{d}\left(F, F^{(1)}\right) \leq \frac{\epsilon}{8}
$$

We will show that $F^{(1)}\left(x_{*}\right) \in v\left(F^{(1)}\right)$. Assume that $x \in X$ and that

$$
F^{(1)}(x) \leq F^{(1)}\left(x_{*}\right)=F\left(x_{*}\right) .
$$

By (2.11), (2.5), and (2.6)

$$
F\left(x_{*}\right) \geq F^{(1)}(x)=\psi(x) F\left(x_{*}\right)+(1-\psi(x)) F(x) .
$$

If $\psi(x)=1$, then $F^{(1)}(x)=F\left(x_{*}\right)$. If $\psi(x)<1$, then by (2.12) and (2.1) $F\left(x_{*}\right) \geq F(x)$ and $F\left(x_{*}\right)=F(x)$ and in view of $(2.5)$ and (2.6) $F^{(1)}(x)=F\left(x_{*}\right)$. Therefore $F\left(x_{*}\right) \in v\left(F^{(1)}\right)$. 
4 A density result in vector optimization

For $x \in X$ and $i \in\{1, \ldots, n\}$ set

$$
\begin{gathered}
f_{i}^{(2)}(x)=f_{i}^{(1)}(x)+\min \left\{1, \max \left\{\rho\left(x, x_{*}\right)-\frac{\delta}{2}, 0\right\}\right\}\left(\frac{\epsilon}{8}\right), \\
F^{(2)}=\left(f_{1}^{(2)}, \ldots, f_{n}^{(2)}\right) .
\end{gathered}
$$

Clearly

$$
F^{(2)} \in \mathscr{A}, \quad F^{(2)}(x) \geq F^{(1)}(x) \quad \forall x \in X
$$

By (2.13), and (2.7)

$$
F^{(2)}(x)=F^{(1)}(x)=F\left(x_{*}\right) \quad \forall x \in X \text { satisfying } \rho\left(x, x_{*}\right) \leq \frac{\delta}{2} .
$$

By (2.13) and (2.7) for each $x \in X$ satisfying $\rho\left(x, x_{*}\right) \in[\delta / 2, \delta]$,

$$
F^{(2)}(x)=F^{(1)}\left(x_{*}\right)+\left(\frac{\epsilon}{8}\right)\left[\rho\left(x, x_{*}\right)-\frac{\delta}{2}\right](1,1, \ldots, 1) .
$$

By (2.13)

$$
\tilde{d}\left(F^{(2)}, F^{(1)}\right) \leq \frac{\epsilon}{8} .
$$

This inequality and (2.10) imply that

$$
\tilde{d}\left(F, F^{(2)}\right) \leq \frac{\epsilon}{4} .
$$

It is clear that the inclusion $F\left(x_{*}\right) \in v\left(F^{(1)}\right),(2.15)$, and (2.16) imply that

$$
F\left(x_{*}\right) \in v\left(F^{(2)}\right) .
$$

We will show that

$$
\text { if } x \in X \text { satisfies } F^{(2)}(x)=F\left(x_{*}\right) \text {, then } \rho\left(x, x_{*}\right) \leq \frac{\delta}{2} .
$$

Assume that

$$
x \in X, \quad F^{(2)}(x)=F\left(x_{*}\right) .
$$

In view of (2.15) and (2.22) $F^{(1)}(x) \leq F^{(2)}(x) \leq F\left(x_{*}\right)$. Together with the inclusion $F\left(x_{*}\right) \in v\left(F^{(1)}\right)$ the inequality above implies that $F^{(1)}(x)=F\left(x_{*}\right) \geq F^{(2)}(x)$. Combined with (2.13) this relation implies that

$$
\rho\left(x, x_{*}\right) \leq \frac{\delta}{2}
$$

Thus (2.21) is proved. 
Since $F\left(x_{*}\right) \in v\left(F^{(1)}\right)$, it follows from (2.13) that the following property holds.

(P1) For each $x \in X$ satisfying $\rho\left(x, x_{*}\right)>\delta / 2$ there is $i \in\{1, \ldots, n\}$ such that

$$
f_{i}^{(1)}(x) \geq f_{i}^{(1)}\left(x_{*}\right)=f_{i}\left(x_{*}\right)
$$

and that

$$
\begin{aligned}
f_{i}^{(2)}(x) & =f_{i}^{(1)}(x)+\left(\frac{\epsilon}{8}\right) \min \left\{1, \rho\left(x, x_{*}\right)-\frac{\delta}{2}\right\} \\
& \geq f_{i}^{(1)}\left(x_{*}\right)+\left(\frac{\epsilon}{8}\right) \min \left\{1, \rho\left(x, x_{*}\right)-\frac{\delta}{2}\right\} \\
& =f_{i}\left(x_{*}\right)+\left(\frac{\epsilon}{8}\right) \min \left\{1, \rho\left(x, x_{*}\right)-\frac{\delta}{2}\right\}
\end{aligned}
$$

Choose

$$
\delta_{0} \in\left(0, \frac{\delta}{8}\right), \quad \lambda_{0} \in\left(0, \frac{\epsilon}{16}\right)
$$

Define functions $\phi_{1}, \phi_{2}:[0, \infty) \rightarrow R$ as follows:

$$
\begin{gathered}
\phi_{1}(x)=x, \quad x \in[0,1], \quad \phi_{1}(x)=1, \quad x \in(1,2], \\
\phi_{1}(x)=x-1, \quad x \in(2,8], \quad \phi_{1}(x)=15-x, \quad x \in(8,14], \\
\phi_{1}(x)=1, \quad x \in(14,15], \quad \phi_{1}(x)=16-x, \quad x \in(15,16], \quad \phi_{1}(x)=0, \quad x \in(16, \infty), \\
\phi_{2}(x)=-x, \quad x \in[0,2], \quad \phi_{2}(x)=x-4, \quad x \in(2,8], \\
\phi_{2}(x)=12-x, \quad x \in(8,14], \quad \phi_{2}(x)=-16+x, \quad x \in(14,16], \\
\phi_{2}(x)=0, \quad x \in(16, \infty) .
\end{gathered}
$$

It is clear that $\phi_{1}, \phi_{2}$ are continuous functions and that

$$
\sup \left\{\left|\phi_{i}(x)\right|: x \in R, i=1,2\right\} \leq 7 .
$$

Define a function $G=\left(g_{1}, \ldots, g_{n}\right): X \rightarrow R^{n}$ as follows:

$$
g_{1}(x)=f_{1}^{(2)}(x)+\lambda_{0} \phi_{1}\left(16 \rho\left(x, x_{*}\right) \delta_{0}^{-1}\right), \quad x \in X ;
$$

for $i \in\{2, \ldots, n\}$

$$
g_{i}(x)=f_{i}^{(2)}(x)+\lambda_{0} \phi_{2}\left(16 \rho\left(x, x_{*}\right) \delta_{0}^{-1}\right), \quad x \in X
$$

Clearly, $G \in \mathscr{A}$. By (2.31), (2.30), (2.29), and (2.26) $\tilde{d}\left(G, F^{(2)}\right) \leq \epsilon / 2$. Together with (2.19) this implies that

$$
\tilde{d}(G, F)<\epsilon .
$$


6 A density result in vector optimization

Relations (2.30), (2.31), and (2.28) imply that for each $x \in X$ satisfying $\rho\left(x, x_{*}\right) \geq \delta_{0}$

$$
G(x)=F^{(2)}(x)
$$

In view of (2.33) and (2.16) for each $x \in X$ satisfying $\rho\left(x, x_{*}\right) \in\left[\delta_{0}, \delta / 2\right]$ we have

$$
G(x)=F\left(x_{*}\right)
$$

It follows from (2.33) and (2.17) that for each $x \in X$ satisfying $\rho\left(x, x_{*}\right) \in[\delta / 2, \delta]$

$$
G(x)=F\left(x_{*}\right)+\left(\frac{\epsilon}{8}\right)\left[\rho\left(x, x_{*}\right)-\frac{\delta}{2}\right](1,1, \ldots, 1) .
$$

By (2.30), (2.31), (2.16), (2.27), and (2.28) for each $x \in X$ satisfying $\rho\left(x, x_{*}\right) \leq \delta_{0} / 16$ we have

$$
g_{1}(x)=f_{1}^{(2)}(x)+\lambda_{0} \rho\left(x, x_{*}\right) \delta_{0}^{-1} 16=f_{1}\left(x_{*}\right)+\lambda_{0} \rho\left(x, x_{*}\right) \delta_{0}^{-} 16
$$

for $i=2, \ldots, n$

$$
\begin{gathered}
g_{i}(x)=f_{i}^{(2)}\left(x_{*}\right)-\lambda_{0} \rho\left(x, x_{*}\right) \delta_{0}^{-1} 16=f_{i}\left(x_{*}\right)-\lambda_{0} \rho\left(x, x_{*}\right) \delta_{0}^{-1} 16, \\
G(x)=F\left(x_{*}\right)+\lambda_{0} \rho\left(x, x_{*}\right) \delta_{0}^{-1} 16(1,-1, \ldots,-1) .
\end{gathered}
$$

Relations (2.27), (2.30), (2.31), (2.16), and (2.28) imply that for each $x \in X$ satisfying $\rho\left(x, x_{*}\right)=\delta_{0} / 8$ we have

$$
g_{1}(x)=f_{1}^{(2)}(x)+\lambda_{0} \phi_{1}(2)=f_{1}\left(x_{*}\right)+\lambda_{0}
$$

for $i=2, \ldots, n$

$$
\begin{aligned}
& g_{i}(x)=f_{i}^{(2)}(x)+\lambda_{0} \phi_{2}(2)=f_{i}\left(x_{*}\right)-2 \lambda_{0}, \\
& G(x)=F\left(x_{*}\right)+\lambda_{0}(1,-2,-2, \ldots,-2) .
\end{aligned}
$$

We will show that for each $x \in X$ satisfying $\rho\left(x, x_{*}\right) \leq \delta_{0}$ the following property holds.

(P2) There is $z \in X$ such that $\rho\left(z, x_{*}\right) \in\left[0, \delta_{0} / 16\right] \cup\left\{\delta_{0} / 8\right\}$ and $G(z) \leq G(x)$.

Let $x \in X$ satisfy

$$
\rho\left(x, x_{*}\right) \leq \delta_{0}
$$


Clearly, if $\rho\left(x, x_{*}\right) \leq \delta_{0} / 16$, then (P2) holds with $z=x$. We consider the following cases:

$$
\begin{aligned}
& \rho\left(x, x_{*}\right) \in\left[\frac{\delta_{0}}{16}, \frac{\delta_{0}}{8}\right] ; \\
& \rho\left(x, x_{*}\right) \in\left(\frac{\delta_{0}}{8}, \frac{\delta_{0}}{2}\right] ; \\
& \rho\left(x, x_{*}\right) \in\left(\frac{\delta_{0}}{2}, \frac{7}{8} \delta_{0}\right] ; \\
& \rho\left(x, x_{*}\right) \in\left(\frac{7}{8} \delta_{0}, \frac{1}{16} \delta_{0}\right] ; \\
& \rho\left(x, x_{*}\right) \in\left(\frac{15}{16} \delta_{0}, \delta_{0}\right] .
\end{aligned}
$$

Let (2.43) hold. Then by (2.43), (2.30), (2.31), (2.27), (2.28), and (2.16) we have

$$
g_{1}(x)=f_{1}^{(2)}(x)+\lambda_{0} \phi_{1}\left(\rho\left(x, x_{*}\right) \delta_{0}^{-1} 16\right)=f_{1}\left(x_{*}\right)+\lambda_{0}
$$

for $i=2, \ldots, n$

$$
g_{i}(x)=f_{i}^{(2)}(x)+\lambda_{0} \phi_{2}\left(\rho\left(x, x_{*}\right) \delta_{0}^{-1} 16\right)=f_{i}\left(x_{*}\right)-\lambda_{0}\left(\rho\left(x, x_{*}\right) \delta_{0}^{-1} 16\right) \geq f_{i}\left(x_{*}\right)-2 \lambda_{0} .
$$

Together with (2.41) these relations imply that

$$
G(x) \geq G(z) \quad \text { if } z \in X \text { satisfies } \rho\left(z, x_{*}\right)=\frac{\delta_{0}}{8} \text {. }
$$

Thus property (P2) holds if (2.43) is valid.

Assume that (2.44) is true. By (2.44), (2.30), (2.31), (2.27), (2.28), and (2.16) we have

$$
g_{1}(x)=f_{1}\left(x_{*}\right)+\lambda_{0}\left(\rho\left(x, x_{*}\right) \delta_{0}^{-1} 16-1\right) \geq f_{1}\left(x_{*}\right)+\lambda_{0} ;
$$

for $i=2, \ldots, n$

$$
g_{i}(x)=f_{i}\left(x_{*}\right)+\lambda_{0}\left(\rho\left(x, x_{*}\right) \delta_{0}^{-1} 16-4\right) \geq f_{i}\left(x_{*}\right)+\lambda_{0}(-2) .
$$

Together with (2.41) these relations imply that for each $z \in X$ satisfying $\rho\left(z, x_{*}\right)=\delta_{0} / 8$ we have $G(z) \leq G(x)$. Thus property (P2) holds if (2.44) is valid.

Assume that (2.45) holds. By (2.45), (2.30), (2.31), (2.27), (2.28), and (2.16) we have

$$
g_{1}(x)=f_{1}\left(x_{*}\right)+\lambda_{0}\left(15-\rho\left(x, x_{*}\right) \delta_{0}^{-1} 16\right) \geq f_{1}\left(x_{*}\right)+\lambda_{0} ;
$$

for $i=2, \ldots, n$

$$
g_{i}(x)=f_{i}\left(x_{*}\right)+\lambda_{0}\left(12-\rho\left(x, x_{*}\right) \delta_{0}^{-1} 16\right) \geq f_{i}\left(x_{*}\right)-2 \lambda_{0} .
$$


Together with (2.41) these relations imply that for each $z \in X$ satisfying $\rho\left(z, x_{*}\right)=\delta_{0} / 8$ we have $G(z) \leq G(x)$. Thus property (P2) holds if (2.45) is valid.

Assume that (2.46) holds. By (2.46), (2.30), (2.31), (2.27), (2.28), and (2.16) we have

$$
g_{1}(x)=f_{1}\left(x_{*}\right)+\lambda_{0}
$$

for $i=2, \ldots, n$

$$
g_{i}(x)=f_{i}\left(x_{*}\right)+\lambda_{0}\left(-16+\rho\left(x, x_{*}\right) \delta_{0}^{-1} 16\right) \geq f_{i}\left(x_{*}\right)-2 \lambda_{0} .
$$

Together with (2.41) these relations imply that for each $z \in X$ satisfying $\rho\left(z, x_{*}\right)=\delta_{0} / 8$ we have $G(z) \leq G(x)$. Thus property (P2) holds if (2.46) is valid.

Assume that (2.47) holds. By (2.47), (2.30), (2.31), (2.27), (2.28), and (2.16) we have

$$
g_{1}(x)=f_{1}\left(x_{*}\right)+\lambda_{0}\left(16-\rho\left(x, x_{*}\right) \delta_{0}^{-1} 16\right) ;
$$

for $i=2, \ldots, n$

$$
g_{i}(x)=f_{i}\left(x_{*}\right)+\lambda_{0}\left(\rho\left(x, x_{*}\right) \delta_{0}^{-1} 16-16\right) .
$$

Since the space $X$ is connected, it follows from (2.3) and (2.47) that there is $z \in X$ such that

$$
\rho\left(z, x_{*}\right)=\delta_{0}-\rho\left(x, x_{*}\right) \in\left[0, \frac{\delta_{0}}{16}\right] .
$$

In view of (2.59), (2.38), (2.57), and (2.58)

$$
\begin{aligned}
G(z) & =F\left(x_{*}\right)+\lambda_{0} \rho\left(z, x_{*}\right) \delta_{0}^{-1} 16(1,-1, \ldots,-1) \\
& =F\left(x_{*}\right)+\lambda_{0}\left(\delta_{0}-\rho\left(x, x_{*}\right)\right) \delta_{0}^{-1} 16(1,-1,-1, \ldots,-1)=G(x) .
\end{aligned}
$$

Thus property (P2) holds if (2.47) is valid.

We have shown that (P2) holds in all the cases. We have also shown that the following property holds.

(P3) For each $x \in X$ satisfing $\rho\left(x, x_{*}\right) \leq \delta_{0}$ there is $z \in X$ such that

$$
\rho\left(z, x_{*}\right) \in\left[0, \frac{\delta_{0}}{16}\right] \cup\left\{\frac{\delta_{0}}{8}\right\}, \quad G(z) \leq G(x) .
$$

We will show that the following property holds.

(P4) If $x \in X$ satisfies $\rho\left(x, x_{*}\right) \geq \delta / 2$ and $z \in X$ satisfies $\rho\left(z, x_{*}\right) \in\left\{\delta_{0} / 8\right\} \cup\left[0, \delta_{0} / 16\right]$, then the inequality $G(x) \leq G(z)$ does not hold.

Assume that

$$
x \in X, \quad z \in X, \quad \rho\left(x, x_{*}\right)>\frac{\delta}{2}, \quad \rho\left(z, x_{*}\right) \in\left\{\frac{\delta_{0}}{8}\right\} \cup\left[0, \frac{\delta_{0}}{16}\right] .
$$

By property (P1) and (2.62) there is $j \in\{1, \ldots, n\}$ such that

$$
f_{j}^{(2)}(x) \geq f_{j}\left(x_{*}\right)+\left(\frac{\epsilon}{8}\right) \min \left\{1, \rho\left(x, x_{*}\right)-\frac{\delta}{2}\right\} .
$$


By (2.13) and (2.62)

$$
f_{i}^{(2)}(x)>f_{i}\left(x_{*}\right), \quad i=1, \ldots, n .
$$

Together with (2.33) and (2.26) this implies that

$$
g_{i}(x)>f_{i}\left(x_{*}\right), \quad i=1, \ldots, n .
$$

It follows from (2.62) that

$$
\begin{aligned}
G(z) & \in\left\{F\left(x_{*}\right)+\lambda_{0}(1,-1,-1, \ldots,-1) t: t \in[0,1]\right\} \\
\cup & \left\{F\left(x_{*}\right)+\lambda_{0}(1,-2,-2, \ldots,-2)\right\} .
\end{aligned}
$$

It follows from this inclusion and (2.65) that $G(x) \leq G(z)$ does not hold. Therefore property (P4) holds.

Let $t \in[0,1)$. We show that $F\left(x_{*}\right)+\lambda_{0} t(1,-1,-1, \ldots,-1) \in v(G)$. Since the space $X$ is connected, it follows from (2.3) that there is $z \in X$ such that

$$
\rho\left(z, x_{*}\right)=\frac{\delta_{0}}{16} t
$$

By (2.67) and (2.38)

$$
\begin{aligned}
G(z) & =F\left(x_{*}\right)+\lambda_{0} \rho\left(z, x_{*}\right) \delta_{0}^{-1} 16(1,-1,-1, \ldots,-1) \\
& =F\left(x_{*}\right)+t \lambda_{0}(1,-1,-1, \ldots,-1) \in G(X) .
\end{aligned}
$$

Assume that

$$
x \in X, \quad G(x) \leq G(z) .
$$

We will show that $G(x)=G(z)$. If $\rho\left(x, x_{*}\right)>\delta / 2$, then by (2.13), (2.33), and (2.26) the relation (2.65) is true and together with (2.69) this implies that $G(z) \gg F\left(x_{*}\right)$. This contradicts (2.68). Therefore

$$
\rho\left(x, x_{*}\right) \leq \frac{\delta}{2}
$$

If $\rho\left(x, x_{*}\right) \in\left[\delta_{0}, \delta / 2\right]$, then by (2.34), (2.69), (2.68), and (2.67)

$$
G(x)=F\left(x_{*}\right), \quad t=0, \quad z=x_{*}, \quad G(z)=G(x) .
$$

Assume that

$$
\rho\left(x, x_{*}\right) \leq \delta_{0} .
$$

By (2.72) and property (P3) there is $y \in X$ such that

$$
G(y) \leq G(x), \quad \rho\left(y, x_{*}\right) \in\left[0, \frac{\delta_{0}}{16}\right] \cup\left\{\frac{\delta_{0}}{8}\right\} .
$$


In view of (2.73), (2.69), and (2.68)

$$
G(y) \leq G(x) \leq G(z)=F\left(x_{*}\right)+\lambda_{0} t(1,-1,-1, \ldots,-1) .
$$

If $\rho\left(y, x_{*}\right)=\delta_{0} / 8$, then by $(2.41)$

$$
G(y)=F\left(x_{*}\right)+\lambda_{0}(1,-2,-2, \ldots,-2)
$$

and since $t \in[0,1)$, the equality above contradicts (2.74). Therefore in view of (2.73)

$$
\rho\left(y, x_{*}\right) \in\left[0, \frac{\delta_{0}}{16}\right]
$$

By (2.76) and (2.38)

$$
G(y)=F\left(x_{*}\right)+\lambda_{0} \rho\left(x, x_{*}\right) \delta_{0}^{-1}(16)(1,-1,-1, \ldots,-1) .
$$

Together with (2.74) this equality implies that $t=\rho\left(x, x_{*}\right) \delta_{0}^{-1} 16$ and $G(y)=G(x)=G(z)$. Thus we have shown that (2.69) implies that $G(x)=G(z)$. Therefore

$$
F\left(x_{*}\right)+\lambda_{0} t(1,-1,-1, \ldots,-1) \in v(G) \quad \forall t \in[0,1) .
$$

Let $x \in X$ satisfy $\rho\left(x, x_{*}\right)=\delta_{0} / 8$. (Note that by (2.3) such an $x$ exists.) In view of (2.41)

$$
G(x)=F\left(x_{*}\right)+\lambda_{0}(1,-2,-2, \ldots,-2)<F\left(x_{*}\right)+\lambda_{0}(1,-1,-1, \ldots,-1) .
$$

Thus

$$
F\left(x_{*}\right)+\lambda_{0}(1,-1,-1, \ldots,-1) \notin v(G) .
$$

Together with (2.78) this implies that $v(G)$ is not closed. Theorem 1.2 is proved.

\section{References}

[1] G.-Y. Chen, X. Huang, and X. Yang, Vector Optimization, Lecture Notes in Economics and Mathematical Systems, vol. 541, Springer, Berlin, 2005.

[2] J. P. Dauer and R. J. Gallagher, Positive proper efficient points and related cone results in vector optimization theory, SIAM Journal on Control and Optimization 28 (1990), no. 1, 158-172.

[3] M. Ehrgott and X. Gandibleux (eds.), Multiple Criteria Optimization: State of the Art Annotated Bibliographic Surveys, International Series in Operations Research \& Management Science, vol. 52, Kluwer Academic, Massachusetts, 2002.

[4] J. Jahn, Vector Optimization. Theory, Applications, and Extensions, Springer, Berlin, 2004.

[5] T. Tanino, Stability and sensitivity analysis in convex vector optimization, SIAM Journal on Control and Optimization 26 (1988), no. 3, 521-536.

[6] A. J. Zaslavski, A generic result in vector optimization, preprint.

Alexander J. Zaslavski: Department of Mathematics, The Technion-Israel Institute of Technology, 32000 Haifa, Israel

E-mail address: ajzasl@tx.technion.ac.il 


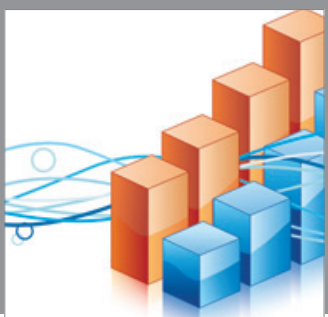

Advances in

Operations Research

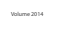

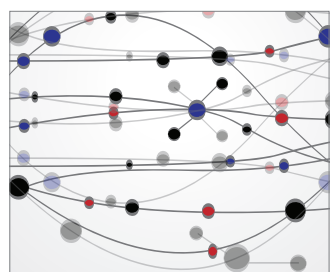

\section{The Scientific} World Journal
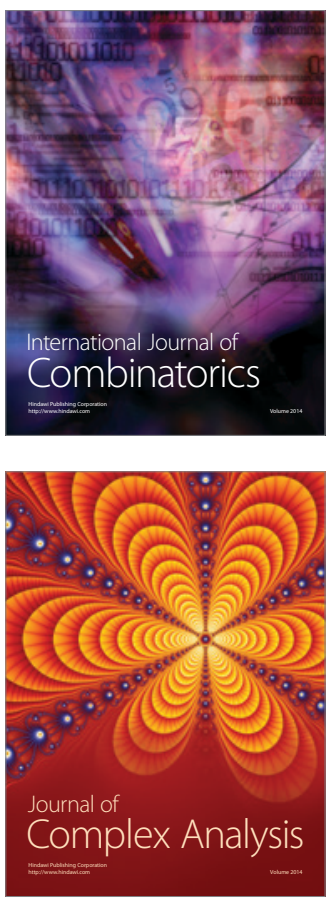

International Journal of

Mathematics and

Mathematical

Sciences
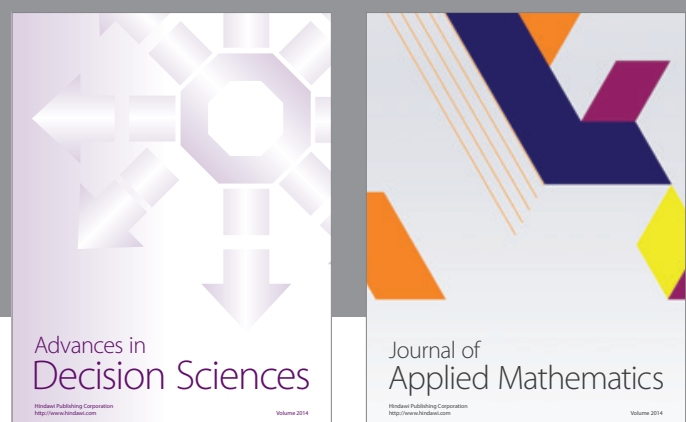

Journal of

Applied Mathematics
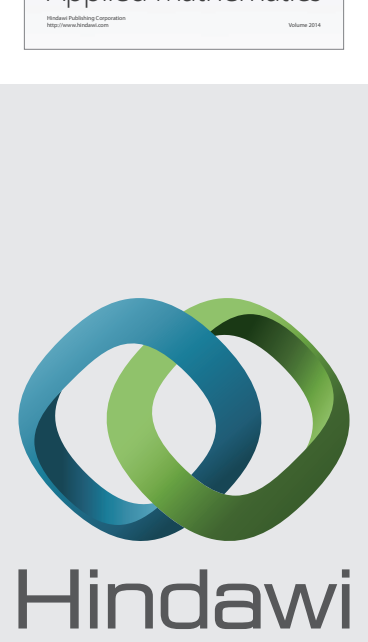

Submit your manuscripts at http://www.hindawi.com
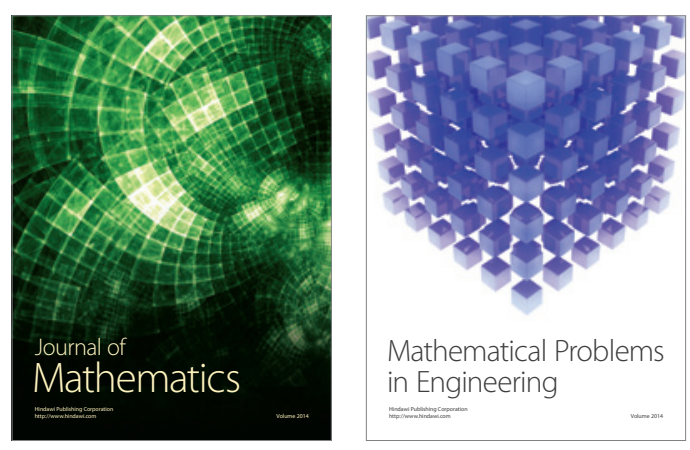

Mathematical Problems in Engineering
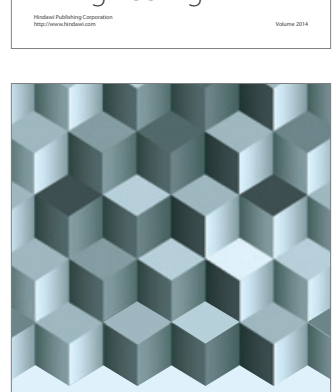

Journal of

Function Spaces
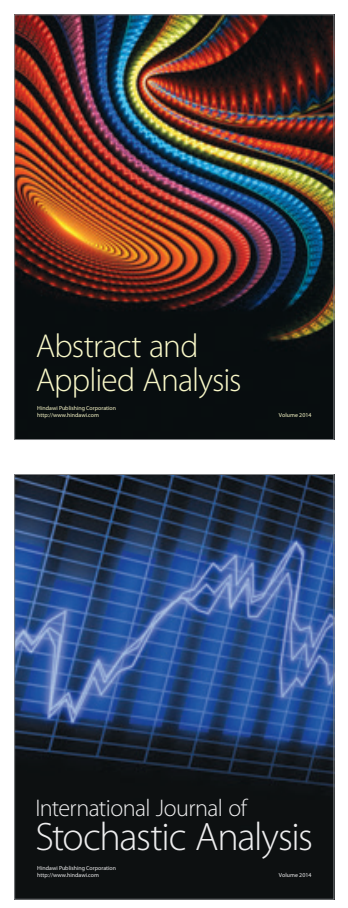

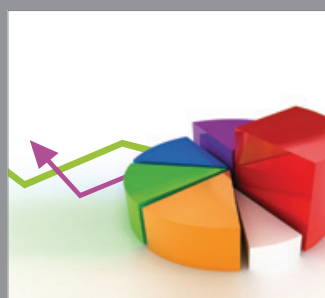

ournal of

Probability and Statistics

Promensencen
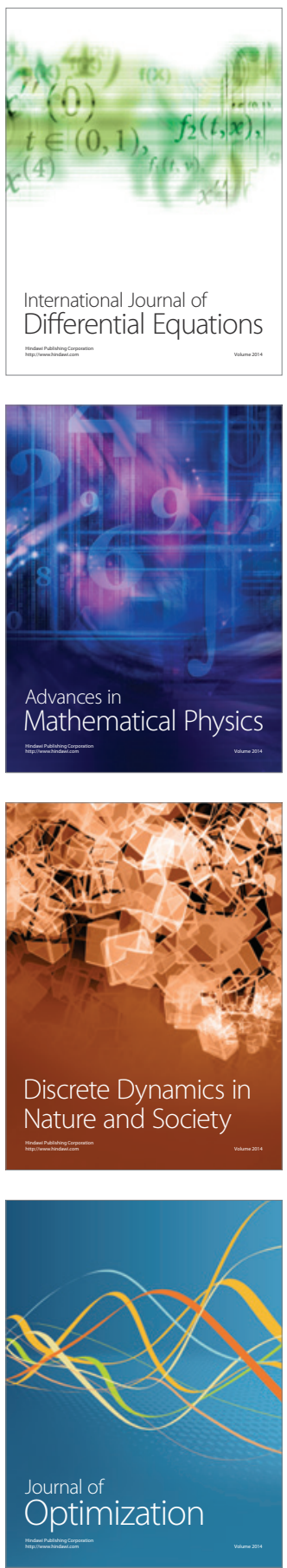